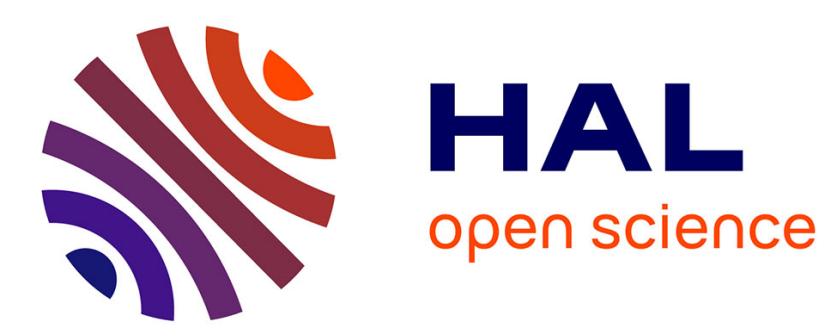

\title{
PHÉNOMĖNES DE TRANSPORT DANS LES SOLUTIONS DE POLYÉLECTROLYTES
}

\author{
Anne Schmitt, R. Varoqui, J. Meullenet
}

\section{To cite this version:}

Anne Schmitt, R. Varoqui, J. Meullenet. PHÉNOMÈnES DE TRANSPORT DANS LES SOLUTIONS DE POLYÉLECTROLYTES. Journal de Physique Colloques, 1978, 39 (C2), pp.C2-161-C2168. 10.1051/jphyscol:1978228 . jpa-00217383

\section{HAL Id: jpa-00217383 https://hal.science/jpa-00217383}

Submitted on 1 Jan 1978

HAL is a multi-disciplinary open access archive for the deposit and dissemination of scientific research documents, whether they are published or not. The documents may come from teaching and research institutions in France or abroad, or from public or private research centers.
L'archive ouverte pluridisciplinaire HAL, est destinée au dépôt et à la diffusion de documents scientifiques de niveau recherche, publiés ou non, émanant des établissements d'enseignement et de recherche français ou étrangers, des laboratoires publics ou privés. 


\title{
PHÉNOMĖNES DE TRANSPORT DANS LES SOLUTIONS DE POLYÉLECTROLYTES
}

\author{
A. SCHMITT, R. VAROQUI et J. P. MEULLENET \\ Centre de Recherches sur les Macromolécules, \\ C.N.R.S., 6, rue Boussingault, 67083 Strasbourg Cedex, France
}

\begin{abstract}
Résumé. - Les processus dissipatifs au sein de solutions polyélectrolytiques constituent un ensemble de phénomènes très riche, tant par sa variété que par le nombre de paramètres d'étude intervenant. Nous limitons notre analyse à deux situations-limites, celles de solutions en absence ou avec excès d'électrolyte ordinaire.

Le formalisme utilisé est celui de la thermodynamique des processus irréversibles linéaires et les différents coefficients de transport (diffusion, autodiffusion, sédimentation, conductibilité...) sont exprimés en fonction de coefficients de frottement binaires. La signification de ces coefficients de frottement est analysée en établissant le lien avec les classiques effets de relaxation et électrophorétique, et l'on souligne la profonde interdépendance entre interactions hydrodynamiques et électrostatiques.

Nous montrons comment les coefficients de frottement peuvent être calculés à partir de données expérimentales ou à partir de théories liées à des modèles. La discussion des résultats obtenus conduit à des conclusions intéressantes concernant :

- le phénomène de condensation ionique,

- la signification de l'effet de charge en sédimentation et diffusion,

- la validité des relations de Svedberg et de Nernst-Einstein.

Enfin, la considération de certains résultats théoriques et expérimentaux suggère que l'aspect dynamique des fluctuations de concentration joue sans doute un rôle important, négligé jusqu'à présent dans de nombreuses approches théoriques.
\end{abstract}

Abstract. - The study of irreversible processes in polyelectrolyte solutions covers a great variety of experimental situations and parameters. We limit the analysis to salt-free and excess-of-salt conditions.

The formalism of linear irreversible thermodynamics is used to express transport coefficients (diffusion, self-diffusion, sedimentation, conductivity...) as a function of binary friction coefficients. We discuss the significance of these friction coefficients, in relation with the classical relaxation and electrophoretic effects, and the correlation between hydrodynamic and electrostatic interactions is emphasized.

We show how friction coefficients can be obtained from experimental data or computed using molecular theories. The obtained results lead to some interesting conclusions concerning :

- the ion-condensation phenomena;

- the significance of the charge effect in sedimentation and diffusion;

- the validity of the Svedberg and Nernst-Einstein relations.

Theoretical and experimental results suggest that the dynamical aspect of ion concentration fluctuations plays a major role, neglected up until now in most theoretical approaches.

1. Introduction. - Il est à présent bien connu que les propriétés d'équilibre des solutions de polyélectrolytes linéaires (pression osmotique, chaleur de dilution, activités ioniques...) sont peu sensibles à la conformation globale du polyion [1]. Au voisinage et au-delà du seuil de condensation ionique, ces propriétés sont décrites de manière quantitative en assimilant le polyion à une ligne portant une distribution de charge uniforme. Il en résulte une atmo- sphère ionique à symétrie cylindrique, et l'énergie libre associée à un tel modèle a été évaluée et discutée par plusieurs auteurs [2, 3]. Ainsi, pour les propriétés thermodynamiques évoquées, des paramètres aussi importants que la concentration en polymère ou le degré de polymérisation deviennent secondaires.

Lorsqu'on aborde l'étude des processus dissipatifs, il devient évident que les paramètres de transport caractérisant le polyion dépendent de sa confor- 
mation. Toutefois, ils dépendent aussi de la présence d'une atmosphère ionique, et l'on voit apparaître la convergence de concepts développés dans l'étude des polymères neutres et dans celle des électrolytes ordinaires. Cette convergence nous fait pressentir la richesse potentielle de ces recherches ainsi que leur complexité. Elles s'inscrivent néanmoins dans un contexte encourageant où les études conformationnelles connaissent un nouveau développement [4].

Dans le cadre du présent article, il n'est pas possible de présenter une discussion détaillée des problèmes évoqués. Aussi limiterons-nous notre investigation à quelques aspects qui nous paraissent significatifs par les problèmes qu'ils posent et les perspectives qu'ils offrent, à savoir :

a) la transposition, pour l'étude des polyélectrolytes, des concepts d'effet de relaxation et d'effet électrophorétique, désormais bien connus en théorie des électrolytes ordinaires [5];

b) la détermination expérimentale des paramètres de transport et les conséquences du phénomène de condensation ionique ;

c) l'effet de charge en sédimentation et diffusion;

d) les prévisions contradictoires des théories appliquées à des modèles de polyélectrolytes.

Le formalisme que nous utilisons est celui de la thermodynamique des processus irréversibles linéaires. Il est suffisamment général pour ne pas dépendre d'un modèle particulier de solution polyélectrolytique et il s'impose dans la mesure où les effets de couplage sont, comme nous le verrons, importants.

2. Définitions et paramètres d'étude. - Soit une solution d'un polyélectrolyte monodisperse, de concentration $C_{\mathrm{p}}$, contenant éventuellement un électrolyte ordinaire, en concentration $C_{\sigma}$, dans un solvant repéré par l'indice $« w »$. Pour simplifier, nous supposerons les ions ordinaires monovalents et noterons $z_{1}, z_{2}$ et $z_{3}$ les valences (algébriques) respectives des contreions, coions et polyions. Les conditions d'électroneutralité entraînent

$$
\begin{array}{r}
z_{1}+z_{2}=0 \\
z_{1} v+z_{3}=0
\end{array}
$$

$v$ est le nombre de contreions libres par polyion. Il pourra différer du nombre stœchiométrique $v_{\mathrm{s}}$ et, $s$ 'il en est ainsi, nous noterons $z_{3 \mathrm{~s}}$ la valence stæchiométrique $\left(z_{3} \leqslant z_{3 \mathrm{~s}}\right)$. La composition ionique stæechiométrique de la solution peut être caractérisée par la fraction $x$ de contreions apportés en solution par le polyélectrolyte, à savoir :

$$
x=\frac{v_{\mathrm{s}} C_{\mathrm{p}}}{v_{\mathrm{s}} C_{\mathrm{p}}+C_{o}}
$$

$x$ peut varier continûment de 0 à 1 , mais nous n'envisagerons que les limites $x \sim 1$ (absence de sel, soit
$C_{\sigma} \ll v_{\mathrm{s}} C_{\mathrm{p}}$ ) et $x \sim 0$ (excès de sel, $C_{\sigma} \gg v_{\mathrm{s}} C_{\mathrm{p}}$ ), limites pour lesquelles les formulations restent relativement simples.

3. Le formalisme. - Nous ne considérons ici que les processus de transport dans un milieu isotherme, en équilibre mécanique, excluant par là les propriétés de viscosité analysées par C. Wolff [6]. Exprimons la force thermodynamique généralisée appliquée à l'espèce " $i$ » en fonction des forces de frottement généralisées, proportionnelles aux vitesses moyennes relatives entre substances mobiles [7] :

$$
\nabla\left(-\tilde{\mu}_{i}\right)=\sum_{j \neq i} f_{i j}\left(\mathbf{v}_{i}-\mathbf{v}_{j}\right) \quad(i, j=1,2,3, w)
$$

$\tilde{\mu}_{i}$ est le potentiel électrochimique généralisé de la substance « $i », \mathbf{v}_{i}$ sa vitesse moyenne dans le référentiel du solvant et $f_{i j}$ le coefficient de frottement binaire avec les particules (ions, atomes, molécules) de type « $j »$. Les relations de symétrie d'Onsager s'écrivent :

$$
C_{i} f_{i j}=C_{j} f_{j i}
$$

alors que la condition d'équilibre mécanique s'exprime par une relation de Gibbs-Duhem généralisée :

$$
\sum_{i} C_{i} \nabla\left(-\tilde{\mu}_{i}\right)=0 \quad(i=1,2,3, w) .
$$

Ceci montre que seules trois équations du type (3.1) sont indépendantes. Incidemment, ce formalisme peut être généralisé aux situations où il n'y a pas équilibre mécanique [8].

\section{Solutions de polyélectrolytes en absence de sel} $(x \sim 1)$. -4.1 EFFET DE RFLAXATION ET EFFET ÉLECTROPHORÉTIQUE. SIGNIFICATION DES COEFFICIENTS DE FROTTEMENT BINAIRES. - Considérons tout d'abord une solution diluée de polymères quasiment neutres, chaque macromolécule ne portant qu'une charge élémentaire $e$. Dans un champ électrique d'intensité $\mathbf{E}$, les macromolécules se déplacent avec une vitesse moyenne $\mathbf{v}_{3}^{0}$ telle que

$$
e \mathbf{E}=f_{3 w}^{0} \cdot \mathbf{v}_{3}^{0} .
$$

Supposons alors qu'il soit possible d'ioniser le polymère, sans modifier sa conformation en solution. Chaque polyion porte alors la charge $z_{3} e$ et la solution s'enrichit par unité de volume de $v C_{\mathrm{p}}$ contreions. L'équation (4.1), qui traduit l'équilibre entre force électrique appliquée et force de frottement hydrodynamique s'écrit alors :

$$
z_{3} e(\mathbf{E}-\Delta \mathbf{E})=\left(f_{3 w}^{0}+\delta f_{3 w}\right) \mathbf{v}_{3}=f_{3 w} \mathbf{v}_{3}
$$

$\Delta \mathbf{E}$ est ce qu'on appelle l'intensité du champ de relaxation, proportionnelle à $\mathbf{E}$. En effet, le champ agissant en moyenne sur le polyion est inférieur au champ macroscopique appliqué, en raison notamment de la polarisation de l'atmosphère ionique. Par ailleurs, $\delta f_{3 w}$ exprime l'incrément de frottement 
hydrodynamique dû à la présence de l'atmosphère ionique. On s'attend, par référence à la théorie des électrolytes ordinaires [5] à ce qu'il soit positif, conduisant à un coefficient de frottement hydrodynamique $f_{3 w}$ du polyion supérieur à celui, $f_{3 w}^{0}$, du polymère neutre ayant même conformation.

Il est possible d'établir, à partir de (3.1) et (4.2) une expression du champ de relaxation relatif :

$$
z_{3} e \frac{\Delta \mathbf{E}}{\mathbf{E}}=f_{31}\left(u_{3}-u_{1}\right)
$$

où $u_{i}$ est la mobilité électrophorétique, ou vitesse moyenne par unité de champ appliqué :

$$
u_{i}=\frac{\mathbf{v}_{i}}{\mathbf{E}} .
$$

Les conditions d'électroneutralité (2.1) et de symétrie (3.2) montrent que le même champ de relaxation agit sur les contreions et l'on peut donc écrire, par analogie avec (4.2) :

$$
u_{1}=\frac{e z_{1}}{f_{1 w}}\left(1-\frac{\Delta \mathbf{E}}{\mathbf{E}}\right) .
$$

Connaissant l'expression des mobilités $u_{i}(i=1,3)$ en termes de coefficients de frottement [9], on montre que le champ de relaxation relatif est de la forme :

$$
\frac{\Delta \mathbf{E}}{\mathbf{E}}=\frac{r_{13}}{r_{13}+\bar{r}_{w}}
$$

$r_{13}$ est un coefficient de frottement interionique réduit

$$
r_{13}=\frac{f_{31}}{v C_{\mathrm{p}}}=\frac{f_{13}}{C_{\mathrm{p}}}=r_{31}
$$

et $\bar{r}_{w}$ un coefficient hydrodynamique réduit moyen

$$
\bar{r}_{w}=\frac{f_{3 w} f_{1 w}}{C_{\mathrm{p}}\left(f_{3 w}+v f_{1 w}\right)} .
$$

L'intérêt de l'équation (4.6) est double :

a) $r_{13}$ étant le terme dominant, elle illustre clairement le fait que les coefficients de frottement interioniques $f_{13}$ (ou $f_{31}$ ) traduisent des interactions de type électrostatique, à longue portée.

b) La présence du coefficient $\bar{r}_{w}$, plus lentement variable avec $z_{3}$, montre que le champ de relaxation dépend aussi de paramètres hydrodynamiques.

On voit ainsi qu'interactions électrostatiques et hydrodynamiques concourent de manière complexe à la détermination des coefficients de transport.

4.2 Détermination eXPÉRIMENTALe DeS COEFFICIENTS DE FROTTEMENT. - Pour déterminer expérimentalement les coefficients $f_{1 w}, f_{3 w}$ et $f_{13}$, il suffit d'effectuer trois mesures indépendantes convena- blement choisies Cela est vrai dès lors que l'on connaît sans ambiguïté la valence effective du polyion, soit $z_{3}=z_{3 s}$. Nous donnons ci-dessous l'expression des principaux paramètres de transport mesurables, celle des mobilités en champ électrique ayant déjà été donnée (cf. (4.2), (4.5) et (4.6)). Pour plus de détails se reporter à [9] :

a) nombre de transport des ions, ou fraction de courant électrique transporté par chaque espèce ionique

$$
t_{1}=\frac{f_{3 w}}{f_{\mathrm{p} w}}, \quad t_{3}=\frac{v f_{1 w}}{f_{\mathrm{p} w}}
$$

$f_{\mathrm{pw}}$ est le coefficient de frottement hydrodynamique du polyélectrolyte (polyion + contreions libres) :

$$
f_{\mathrm{p} w}=f_{3 w}+v f_{1 w} ;
$$

b) conductibilité équivalente de la solution, définie en fonction des mobilités par :

$$
\Lambda=e \frac{z_{1}}{\left|z_{1}\right|}\left(u_{1}-u_{3}\right)
$$

d'où, d'après (4.3) et (4.6) :

$$
\Lambda=\frac{e^{2}\left|z_{1}\right|}{f_{13}+C_{\mathrm{p}} \bar{r}_{w}}
$$

c) coefficients de diffusion et de sédimentation :

$$
D_{\mathrm{p}}=\frac{k T v \varphi_{\mathrm{p}}}{f_{\mathrm{p} w}}, \quad S_{\mathrm{p}}=\frac{\bar{M}_{\mathrm{p}}}{f_{\mathrm{p} w}}
$$

$\varphi_{\mathrm{p}}$ est un coefficient thermodynamique peu différent du coefficient osmotique [9] et $\bar{M}_{\mathrm{p}}$ est la masse apparente du polyélectrolyte, compte tenu de la poussée d'Archimède; $k$ est la constante de Boltzmann, $T$ la température.

On peut vérifier aisément que seules deux mesures en champ électrique sont indépendantes, et que sédimentation et diffusion conduisent au même coefficient de frottement, tout en nécessitant une mesure additionnelle $\left(\bar{M}_{\mathrm{p}}\right.$ ou $\left.\varphi_{\mathrm{p}}\right)$. Supposons par exemple que l'on ait mesuré $S_{\mathrm{p}}\left(\right.$ et $\left.\bar{M}_{\mathrm{p}}\right), t_{1}$ et $\Lambda$. Les coefficients $f_{i j}$ s'obtiennent alors par les relations :

$$
\begin{aligned}
& f_{3 w}=t_{1} \frac{\bar{M}_{\mathrm{p}}}{S_{\mathrm{p}}} \\
& f_{1 w}=\frac{1}{v}\left(1-t_{1}\right) \frac{\bar{M}_{\mathrm{p}}}{S_{\mathrm{p}}} \\
& f_{13}=-C_{\mathrm{p}} \bar{r}_{w}+\frac{e^{2}\left|z_{1}\right|}{\Lambda} .
\end{aligned}
$$

Avec l'expression des coefficients d'autodiffusion des contreion et polyion s'introduisent deux coefficients de frottement supplémentaires $f_{11}$ et $f_{33}$, 
aisément calculables à partir des données précédentes, du fait des relations d'Einstein généralisées [7, 10] :

$$
D_{1}=\frac{k T}{f_{1 w}+f_{13}+f_{11}}, \quad D_{3}=\frac{k T}{f_{3 w}+f_{31}+f_{33}} .
$$

4.3 La CONDENSATION IONIQUE. - Avec l'étude des polyélectrolytes à forte densité de charge linéaire surgit une difficulté d'interprétation. Pour de telles solutions, il est expérimentalement établi que le nombre de transport stœchiométrique des contreions est négatif $[11,12]$. Or dans l'expression de $t_{1}$ (voir 4.9), le dénominateur $f_{\mathrm{pw}}$ est nécessairement positif, puisqu'il traduit la dissipation d'énergie lors du processus de sédimentation ou de diffusion. On doit donc avoir, pour les expériences évoquées :

$$
f_{3 w}<0
$$

C'est un résultat surprenant, pour lequel nous ne voyons pas d'interprétation hydrodynamique.

Lorsqu'en revanche on établit la distinction entre ions atmosphériques et ions condensés sur la chaîne du polyion, l'ensemble des résultats observés s'interprète classiquement [9].

A priori, il n'est pas évident qu'il faille négliger la contribution des ions condensés aux processus de transport. D'un point de vue hydrodynamique, on comprend assez bien que, ces ions formant une enveloppe ionique assez dense au voisinage de la chaîne polymérique, cette enveloppe soit imperméable au solvant. Si l'on se réfère en effet aux concepts développés par Debye et Bueche [13], la longueur d'écran hydrodynamique est localement très faible. L'ensemble (polyion + contreions condensés) forme donc une entité en hydrodynamique.

Toutefois, les ions condensés possèdent une mobilité dans la vallée d'énergie potentielle entourant le polyion [14], mobilité locale mesurable, par exemple, en RMN [15]. Cette mobilité est à l'origine de la forte polarisabilité du polyion [15], mais notre hypothèse revient à admettre que sa contribution moyenne au transport de courant est négligeable à basse fréquence à l'image de ce qui se passe pour les paires d'ions [16], et plus classiquement, pour les électrons dans les molécules.

Il serait intéressant de pouvoir déterminer directement par l'expérience, le rapport $f=z_{3} / z_{3 s}$, et de vérifier que, comme le prévoit la théorie de la condensation [3],

$$
f=\xi^{-1}
$$

où $\xi$ est un paramètre de charge linéaire sans dimension, proportionnel à la densité linéaire de charge portée par le polyion. Pour cela, nous avons montré qu'il est nécessaire d'effectuer des mesures du coef- ficient d'autodiffusion du polyion [9] et d'admettre les inégalités :

$$
\begin{aligned}
& f_{11} \ll f_{1 w}+f_{13} \\
& f_{33} \ll f_{3 w}+f_{3}
\end{aligned}
$$

L'hypothèse traduite par (4.19a) paraît justifiée si l'on se réfère aux propriétés des solutions d'électrolytes ordinaires [17]. Par contre (4.19b) signifie que l'on néglige les interactions polyion-polyion dans l'interprétation de l'autodiffusion du polyion. Or, des études théoriques [18] et structurales récentes [19, 20] montrent que l'énergie d'interaction entre polyions, dont la portée augmente avec la dilution, est suffisamment importante devant $k T$ pour justifier un modèle cellulaire de solution, comme l'avaient pressenti les pionniers en ce domaine de recherche [2]. Ainsi, l'approche expérimentale proposée [9] paraît problématique. Nous verrons que, dans la limite de l'excès de sel, une autre possibilité expérimentale est offerte pour la détermination de $z_{3}$ au-delà du seuil de condensation.

5. Solutions de polyélectrolytes avec excès de sel $(x \sim 0)$. - Rappelons que les propriétés thermodynamiques d'équilibre et bon nombre de paramètres de transport dépendent peu de la concentration $C_{p}$, pour les solutions polyélectrolytiques pures. Lorsqu'ils en dépendent (viscosité, conductivité équivalente...), il n'existe pas de limite bien établie [21]. D'où l'intérêt de lois limites du type de celles développées par Manning [3], car elles constituent un cadre de référence pour le domaine des concentrations accessibles à l'expérience.

Il en va autrement en présence de sel, où les extrapolations à $C_{\mathrm{p}} \rightarrow 0$ sont possibles, $C_{\sigma}$ étant maintenu constant. Il a été souvent admis que les paramètres de transport ainsi déterminés sont reliés à la conformation des macromolécules par le biais des mêmes relations que celles admises pour les polymères neutres. Dans les paragraphes qui suivent, nous discuterons brièvement de la validité de telles hypothèses.

Auparavant, insistons sur le fait que la condition d'excès de sel signifie $C_{\sigma} \gg v_{\mathrm{s}} C_{\mathrm{p}}$. Elle ne suppose donc rien quant à la valeur de $C_{\mathrm{p}}$, et peut être valable dans les régimes dilué et semi-dilué.

5.1 L'EFFET DE CHARGE EN SÉDIMENTATION ET DIFFUSION. - Lorsqu'une solution polyélectrolytique pure $(x \sim 1)$ est soumise, en absence de courant électrique, à un champ d'ultracentrifugation ou à un champ d'origine chimique (gradient de concentration), contreions et polyions ont tendance à migrer avec des vitesses différentes. La condition de courant nul $\mathbf{v}_{1}=\mathbf{v}_{3}$ reste toutefois satisfaite grâce à l'apparition d'un champ électrique macroscopique dans la cellule de mesure. La circulation de ce champ peut d'ailleurs être mesurée et constitue une différence de potentiel 
de sédimentation ou de diffusion. C'est ce phénomène qui est couramment appelé effet de charge et les théories successives de Svedberg-Pedersen [22], Pedersen [23] et Alexandrowicz-Daniel [24] (notée A-D par la suite) ont montré que le champ électrostatique évoqué décroît et tend vers zéro avec $x$. De plus, la théorie A-D prévoit que le coefficient de sédimentation limite du polyélectrolyte est de la forme :

$$
S_{\mathrm{p}}=\frac{1}{f_{3 w}}\left\{\bar{M}_{3}+v \frac{\left(S_{1}-S_{2}\right)}{\left(f_{1 w}^{a}\right)^{-1}+\left(f_{2 w}^{a}\right)^{-1}}\right\} .
$$

L'indice $\sigma$ réfère aux propriétés en solution saline pure, $\bar{M}_{3}$ est la masse apparente du polyion et les coefficients $S_{i}(i=1,2)$ sont tels que

$$
S_{i}=\frac{\bar{M}_{i}}{f_{i w}^{\sigma}} .
$$

Rappelons que le coefficient de sédimentation du polyélectrolyte en milieu ternaire est l'analogue d'une mobilité, c'est-à-dire une vitesse par unité de champ d'ultracentrifugation, dans un milieu chimiquement homogène :

$$
S_{\mathrm{p}}=\left[\frac{\mathbf{v}_{\mathrm{p}}}{\omega^{2} \mathbf{r}}\right]_{C_{\mathrm{p}}, C_{\sigma}}
$$

$\omega$ est la vitesse de rotation et $\mathbf{r}$ le rayon vecteur normal à l'axe de rotation.

Un calcul rigoureux de $S_{\mathrm{p}}$, ne négligeant pas les interactions de type électrostatique, conduit à la formule suivante [10] :

$S_{\mathrm{p}}=\frac{1}{f_{3}}\left\{\bar{M}_{3}+v \cdot \frac{\left(S_{1}-S_{2}\right)}{\left(f_{1 w}^{\sigma}\right)^{-1}+\left(f_{2 w}^{\sigma}\right)^{-1}}+S_{\sigma}\left(f_{31}+f_{32}\right)\right\}$

$f_{3}$ est le coefficient de frottement total du polyion avec son environnement :

$$
f_{3}=f_{3 w}+f_{31}+f_{32}
$$

et $S_{\sigma}$ le coefficient de sédimentation de l'électrolyte ordinaire

$$
S_{\sigma}=\frac{\bar{M}_{1}+\bar{M}_{2}}{f_{1 w}^{\sigma}+f_{2 w}^{\sigma}}
$$

La comparaison avec l'équation (5.1) suscite deux commentaires :

a) Les deux expressions sont équivalentes si l'on pose $f_{3 i}=0(i=1,2)$, ce qui montre que la théorie A-D néglige les interactions interioniques à longue portée. L'importance relative des coefficients $f_{3 i}$ sera discutée au paragraphe 5.3.

b) Dans (5.1), le terme proportionnel à $\left(S_{1}-S_{2}\right)$ traduit l'effet de charge secondaire. Il s'établit en effet dans la cellule de mesure un champ électrique secon- daire dû à la sédimentation de l'électrolyte ordinaire. Ce champ agit sur le polyion pour augmenter ou réduire sa mobilité. Dans la théorie A-D, il s'annule pour $S_{1}=S_{2}$ (exemple : le chlorure de sodium), alors que notre approche montre que cet effet de sel secondaire s'annule pour $S_{\sigma}=0$ (exemple : chlorure de tétraméthylammonium). Cela apparait clairement lorsque l'on met (5.4) sous la forme :

$$
S_{\mathrm{p}}=\frac{1}{f_{3}}\left\{\bar{M}_{\mathrm{p}}+S_{\sigma}\left(f_{31}+f_{32}-v f_{1 w}^{\sigma}\right)\right\} \text {. }
$$

En diffusion, l'étude de l'effet de charge est à la fois plus simple et plus complexe. Elle est plus complexe par le fait qu'on est en milieu ternaire et que, en toute rigueur, il n'est pas possible de définir un coefficient de diffusion du polyélectrolyte [10]. Ce n'est que lorsque le gradient de potentiel chimique de l'électrolyte peut être négligé $\left(\nabla \mu_{\sigma}^{\mathrm{c}} \simeq 0\right)$ que l'on peut définir le coefficient de diffusion du polyélectrolyte par la relation :

$$
D_{\mathrm{p}}=-\left[\frac{C_{\mathrm{p}} \mathbf{v}_{\mathrm{p}}}{\nabla C_{\mathrm{p}}}\right]_{\mu \delta} .
$$

Dans la limite $x \sim 0$, on montre qu'on obtient [10] :

$$
D_{\mathrm{p}}=\frac{k T}{f_{3}} \text {. }
$$

Cette expression est très voisine de celle donnant le coefficient d'autodiffusion du polyion :

$$
D_{3}=\frac{k T}{f_{3}+f_{33}} .
$$

Lorsqu'on est en régime dilué, il est légitime d'admettre une inégalité du type (4.19), dans la mesure où la portée des interactions entre polyions, imposée par l'électrolyte ordinaire, est nettement inférieure à l'espacement moyen entre polyions, soit

$$
\kappa^{-1} \ll C_{\mathrm{p}}^{-1 / 3}
$$

$\kappa^{-1}$ est la longueur d'écran de Debye-Hückel, définie par

$$
\kappa^{2}=\frac{8 \pi e^{2} C_{\sigma}}{k T \varepsilon}
$$

$\varepsilon$ est la constante diélectrique du solvant. En général, les concentrations salines choisies sont telles que la longueur d'écran est inférieure au rayon de la pelote polymérique, et l'inégalité (5.11) est donc satisfaite en régime dilué. Ainsi peut-on, dans la plupart des situations expérimentales évoquées, admettre

$$
D_{3}=D_{\mathrm{p}} \text {. }
$$

5.2 ElECTROPHORÈSE. - Lorsqu'on applique un champ électrique uniforme à une solution de polyélectrolyte en présence de sel, la mobilité électro- 
phorétique du polyion s'exprime encore par une équation voisine de (4.2), à savoir [25] :

$$
u_{3}=\frac{z_{3} e}{f_{3 w}}\left(1-\frac{\Delta \mathbf{E}_{3}}{\mathbf{E}}\right) \text {. }
$$

Du fait que l'on est dans un milieu à trois composants ioniques, le champ de relaxation $\Delta \mathbf{E}_{i}$ agissant sur chaque type d'ion est différent. Dans la limite d'excès de sel, il vient

$$
u_{3}=\frac{z_{3} e}{f_{3 w}}\left\{1-\frac{f_{31}\left(u_{3}-u_{1}^{\sigma}\right)+f_{32}\left(u_{3}-u_{2}^{\sigma}\right)}{z_{3} e}\right\}
$$

soit encore

$$
u_{3}=\frac{z_{3} e}{f_{3}}\left(1+\frac{u_{1}^{\sigma} f_{31}+u_{2}^{\sigma} f_{32}}{z_{3} e}\right) .
$$

Les mobilités $u_{1}^{\sigma}$ des ions constituant l'électrolyte ordinaire, à la concentration $C_{\sigma}$, sont des paramètres connus. Nous allons, dans le paragraphe qui suit, procéder au calcul des coefficients $f_{i j}$, ce qui nous conduira à une discussion plus détaillée des relations établies en 5.1 et 5.2 .

5.3 Les THÉORIES MOLÉCULAIRES. RELATIONS DE SVEDBERG ET DE NERNST-EINSTEIN. - Rappelons que deux modèles ont été proposés pour l'étude théorique des solutions de polyélectrolytes : celui du bâtonnet (B) et celui de la sphère perméable (SP). Deux remarques méritent d'être faites :

a) Le modèle (SP) n'est pas totalement spécifié par la donnée du rayon $R$ de la sphère contenant une densité constante de monomères. Il faut aussi connaître la loi de variation du rayon de gyration $R_{\mathrm{G}}$ de la macromolécule avec le degré de polymérisation $N$. Or de telles études n'en sont qu'à leur début $[4,18]$.

b) L'utilisation du modèle (B) n'a pas la même signification en hydrodynamique qu'en électrostatique. Pour les polyélectrolytes flexibles en présence de sel, la portée des interactions électrostatiques est souvent plus faible que celle des interactions hydrodynamiques. En conséquence, le modèle $(B)$ peut être réaliste du point de vue énergétique, mais peu adapté du point de vue dynamique.

5.3.1 Calcul de $f_{3 w}$ - Il n'existe pas, à notre connaissance, de théories permettant le calcul du coefficient hydrodynamique $f_{3 w}$ à travers tout le domaine du paramètre de charge. Aux faibles taux de charge, il est légitime d'utiliser les théories hydrodynamiques relatives aux polymères neutres, négligeant par là l'effet électrophorétique. On trouve une étude critique des différentes théories existantes dans un article de Stockmayer [26].

Aux taux de charge plus élevés, il est nécessaire de tenir compte de l'atmosphère ionique et, pour évaluer $f_{3 w}$, on peut utiliser respectivement les théories de Hermans et coll. [27-29] (modèle SP) et de Henry $[30,31]$. Ces auteurs ont en effet calculé une mobilité électrophorétique $u_{3}^{\mathbf{h}}$ en ne considérant que l'aspect hydrodynamique, et en négligeant donc l'effet de relaxation. D'après les équations (4.2) ou (5.14), cette mobilité est donc de la forme :

$$
u_{3}^{\mathrm{h}}=\frac{z_{3} e}{f_{3 w}}
$$

cette formule résulte d'une approximation de type A-D, appliquée à l'électrophorèse.

5.3.2 Calcul de $f_{31}$ et $f_{32}$. - Les coefficients de frottement du polyion avec les ions de son atmosphère ionique peuvent être calculés explicitement à partir de théories sur l'autodiffusion des petits ions dans les solutions polyélectrolytiques [32-35]. Ces théories considèrent un modèle de solution où les polyions sont distribués selon un réseau régulier, et entourés de leur atmosphère ionique. On étudie ainsi le mouvement brownien des ions dans un potentiel périodique, pour obtenir l'abaissement $\rho_{i}$ de leur coefficient d'autodiffusion :

$$
\rho_{i}=\frac{D_{i}}{D_{i}^{\sigma}} \quad(i=1,2)
$$

$D_{i}$ et $D_{i}^{\sigma}$ sont respectivement les coefficients d'autodiffusion dans la solution saline en présence et en absence de polyélectrolyte. Considérons la limite $x \sim 0$, pour laquelle nous pouvons effectuer un développement limité de $\rho_{i}$ [36] :

$$
\rho_{i}=1-k_{i} x+0\left(x^{2}\right) \text {. }
$$

Ce développement peut aussi s'exprimer en fonction de coefficients de frottement de l'ion $i$ avec son environnement, en présence et en absence de polyélectrolyte,

$$
\rho_{i}=\frac{f_{i}^{\sigma}}{f_{i}^{\sigma}+f_{i 3}}
$$

ce qui suppose que l'interaction avec le polyion se traduit essentiellement à travers le terme $f_{i 3}$. Utilisant la relation de symétrie (3.2), il est possible de déduire, à partir de $(5.19)$ et $(5.20)$

$$
f_{3 i}=k_{i} v f_{i}^{\sigma} \text {. }
$$

D'un point de vue qualitatif, on peut dire que $k_{i}$ traduit un effet d'entraînement partiel des ions de l'atmosphère ionique par le polyion, ce qui augmente le coefficient de frottement de ce dernier.

A titre d'illustration, nous donnons ci-dessous les valeurs de $k_{i}$ obtenues par Manning [35] avec un modèle cylindrique de polyélectrolyte, en fonction du paramètre de charge $\xi$ :

$$
\begin{array}{ll}
k_{1}=k_{2}=\xi / 6 & (\xi \leqslant 1) \\
k_{1}=k_{2}=\xi^{-1} / 6 & (\xi \geqslant 1) .
\end{array}
$$


5.3.3 Relations de Svedberg et de Nernst-Einstein. - Il est possible de calculer la masse apparente d'un polymère neutre à partir de mesures de sédimentation et de diffusion : c'est la relation de Svedberg. Dans le cas des polyélectrolytes et en excès de sel, cette relation ne définit en toute rigueur qu'une masse apparente approchée.

$$
\bar{M}_{\mathrm{p}}^{\mathrm{app}}=k T \frac{S_{\mathrm{p}}}{D_{\mathrm{p}}}
$$

et l'on peut exprimer la différence relative entre $\bar{M}_{\mathrm{p}}^{\text {app }}$ et $\bar{M}_{\mathrm{p}}$ au moyen de (5.7):

$r_{M}=\frac{\bar{M}^{\mathrm{app}}-\bar{M}_{\mathrm{p}}}{\bar{M}_{\mathrm{p}}}=S_{\sigma}^{\mathrm{d}} \frac{\left(f_{31}+f_{32}-v f_{1 w}\right)}{\bar{M}_{\mathrm{p}}}$.

Des calculs sur modèles, utilisant les théories moléculaires évoquées, nous ont permis de montrer que $r_{M}$ reste néanmoins faible $[1,37]$ et seules des mesures précises sur un polyélectrolyte monodisperse pourraient mettre en évidence une différence significative.

Une autre relation importante dans le domaine des électrolytes ordinaires, est la relation limite de NernstEinstein. Appliquée aux polyélectrolytes (limite $x \sim 0$ ), elle fournit une valence apparente du polyion :

$$
z_{3}^{\mathrm{app}}=\frac{k T u_{3}}{e D_{\mathrm{p}}}
$$

Nous avons montré dans un travail récent [25] que cette valence apparente était toujours inférieure à (ou voisine de) la valence effective $z_{3}$ du polyion :

$$
z_{3}^{\mathrm{app}} \leqslant z_{3} .
$$

L'égalité est quasiment obtenue lorsque l'atmosphère ionique est traitée dans le cadre de l'approximation de Debye-Hückel. Une importante conséquence de cette inégalité est qu'elle fournit un moyen de tester la théorie de la condensation à partir de mesures d'électrophorèse et de diffusion.
5.4 CORRÉlations SPATIO-TEMPORELles DaNS LES SOLUTIONS DE POLYÉLECTROLYTES. - Dans les théories moléculaires citées au paragraphe précédent, la dynamique du couplage polyion-atmosphère ionique, telle qu'elle peut apparaître à travers les corrélations de fluctuations - est ignorée. Les calculs portent sur une conformation moyenne du polyion à laquelle correspond une distribution ionique moyenne. Or l'existence d'interactions électrostatiques à longue portée doit entraîner de fortes corrélations spatio-temporelles entre polyions et contreions. Cela apparait clairement dans le spectre de la lumière diffusée par une solution de polyélectrolyte, comme l'ont montré des travaux récents $[38,39]$ et le cadre théorique pour l'étude de cette dynamique est en plein développement $[38,40,41]$.

Un exemple de problème posé est le suivant. Considérons comme modèle de polyion, une sphère chargée en surface. Lorsque la sphère est neutre, son coefficient d'autodiffusion est $D_{3}^{0}$. Si on augmente progressivement la valence $z_{3}$, quelle va être la variation de $D_{3}$ ? Dans le cadre des concepts que nous venons de développer, on prédit une chute de $D_{3}$ pour deux raisons :

a) l'apparition de l'effet électrophorétique entraîne $f_{3 w}>f_{3 w}^{0}$

b) l'existence des coefficients de frottement interioniques $f_{3 i}(i=1,2)$ se traduit, comme nous l'avons vu, par un effet d'entraînement partiel des ions de l'atmosphère ionique.

A partir d'un calcul sur ordinateur, Ermark [40] est arrivé à la conclusion inverse, essentiellement parce que le champ fluctuant créé par l'atmosphère ionique augmente la vitesse du processus de divagation du polyion. Un tel entraînement du polyion par son atmosphère ionique est aussi prédit par Stephen [41]. L'existence de ces résultats contradictoires montre que notre compréhension de ces phénomènes complexes demeure parcellaire.

\section{Bibliographie}

[1] Selegny, E., Mandel, M., Strauss, U. P., Polyelectrolytes (D. Reidel, Dordrecht, Holland) 1974.

[2] Katchalsky, A., AleXandrowicz, Z., Kedem, O., Chemical Physics of Ionic Solutions, Conway, B. E. and Barradas, R. G. eds. (Wiley, New York) 1966.

[3] Manning, G. S., J. Chem. Phys. 51 (1969) 924.

[4] a) Pfeuty, P., Velasco, R. M., De Gennes, P. G., J. Physique Lett. 38 (1977) L 5.

b) Pfeuty, P., J. Physique Colloq. 38 (1977).

[5] RÉsiBoIs, P. M. V., Electrolyte Theory (Harper and Row) 1968.

[6] Wolff, C., J. Physique Colloq. 38 (1977).

[7] Laity, R. W., J. Phys. Chem. 63 (1959) 80, J. Chem. Phys. 30 (1959) 682.

[8] Schmitt, A., Craig, J. B., J. Phys. Chem. 81 (1977) 1338.

[9] Schmitt, A., Varoqui, R., J. Chem. Soc. Faraday Trans. II 69 (1973) 1087.
[10] Varoqui, R., Schmitt, A., Biopolymers, 11 (1972) 1119. [11] Huizenga, J. R., Grieger, P. F., Wall, F. T., J. Am. Chem. Soc. 72 (1950) 2636; 72 (1950) 4228.

[12] Jordan, D. O., Kurucsev, T., Martin, M. L., Trans. Faraday Soc., 65 (1969) 606.

[13] Debye, P., Bueche, P., J. Chem. Phys. 16 (1948) 573.

[14] Oosawa, F., Polyelectrolytes (Marcel Dekker, New York) 1971.

[15] Weill, G., J. Physique Colloq. 38 (1977).

[16] Fuoss, R. M., J. Phys. Chem. 79 (1975) 525.

[17] Schmitт; A., Thèse, Université Pasteur, Strasbourg, 1972.

[18] De Gennes, P. G., Pincus, P., Velasco, R. M., Brochard, F., J. Physique 37 (1976) 1461

[19] a) Moan, M., Wolff, C., Ober, R., Polymer 16 (1975) 781.

b) Moan, M., Wol.fF, C., Polymer 16 (1976) 776.

[20] Schaeffer, D. W., ACKerson, B. J., Phys. Rev. Lett. 35 (1975) 1448. 
[21] Armstrong, R. W., Strauss, U. P., Polyelectrolytes in Encyclopedia of Polymer Science and Technology (Wiley, New York) 10 (1969) 1169.

[22] Svedberg, T., Pedersen, K. O., The Ultracentrifuge (Clarendon Press, Oxford) 1940.

[23] Pedersen, K. O., J. Phys. Chem. 62 (1958) 1282.

[24] Alexandrowicz, Z., Daniel, E., Biopolymers 1 (1963) 447, 1 (1963) 473, 6 (1968) 1500.

[25] Schmitr, A., Meullenet, J. P., Varoqui, R., Biopolymers (sous presse).

[26] Stockmayer, W. H., Fluides Moléculaires, Balian, R. et Weill, G., eds. (Gordon and Breach) 1975.

[27] Hermans, J. J., J. Polymer Sci. 18 (1955) 527.

[28] Hermans, J. J., Fujtra, H., Proc. K. Ned. Akad. Wet. B 58 (1955) 182.
[29] Hermans, J. J., Napjus, P. J., J. Colloid Sci. 14 (1959) 252,

[30] Henry, D. C., Proc. R. Soc., Ser A 133 (1931) 106.

[31] Ross, P. D., Biopolymers 2 (1964) 9.

[32] Lifson, S., JACkson, J. L., J. Chem. Phys. 36 (1962) 2410

[33] JaCkson, J. L., Corriell, S. R., J. Chem. Phys. 38 (1963) 959.

[34] BeLl, G. M., Trans. Faraday Soc. 60 (1964) 1752.

[35] Manning, G. S., J. Chem. Phys. 51 (1969) 934.

[36] Schmitr, A., Varoqui, R., Meullenet, J. P., J. Phys. Chem. 81 (1977) 1514

[37] Schmitt, A., Varoqui, R., Eur. Polymer J. 11 (1975) 1, 9.

[38] Berne, B. J., in Photon Correlation Spectroscopy and Velocimetry, Cummins A. Z. and Pike eds. (Plenum Press) 1977.

[39] Meullenet, J. P., Schmitr, A., Candau, S., Chem. Phys. Lett. (sous presse).

[40] Ermak, D. L., J, Chem. Phys. 62 (1975) 4197.

[41] Stephen, M. J., J. Chem. Phys. 55 (1971) 3878. 